\title{
Evaluation of the Prognostic Utility of Computed Tomography New Severity Score in COVID-19 Pneumonia Patients
}

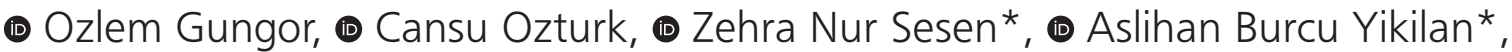 \\ - (1) Selma Uysal Ramadan, (1) Seref Kerem Corbacioglu** \\ University of Health Sciences Turkey, Kecioren Training and Resarch Hospital, Clinic of Radiology, Ankara, Turkey \\ *University of Health Sciences Turkey, Kecioren Training and Research Hospital, Clinic of Infectious Disease, Ankara, Turkey \\ **University of Health Sciences Kecioren Turkey, Training and Research Hospital Clinic of Emergency, Ankara, Turkey
}

\section{Abstract}

\begin{abstract}
Aim: To investigate the relationship between infection markers (lymphocyte, C-reactive protein, and D-dimer) at the time of diagnosis, and the new scoring system created according to the amount and pattern of pneumonic involvement in recent computed tomography (CT) of patients with Coronavirus disease-2019 (COVID-19) pneumonia
\end{abstract}

Methods: We investigated retrospectively patients diagnosed with COVID-19 with positive reverse transcriptase-polymerase chain reaction on throat swabs between March 17 and May 1, 2020. Eighty-nine cases with COVID-19 pneumonia were divided into two groups according to the level of poor prognostic criteria (blood lymphocyte count $<800 / \mu \mathrm{L}$ or C-reactive protein $>10 \mathrm{x}$ upper limit of normal value or D-dimer $>1000 \mathrm{ng} / \mathrm{mL}$ ). The severity of pulmonary parenchymal findings was scored using two separate scoring systems previously as well as a third separate scoring system, namely the "modified CT severity scoring". The cut-off point for severe infection was investigated by comparing the scores of the groups with and without severe infection.

Results: All three scoring systems were significantly higher in the group with severe infection compared to those without severe infections. A modified CT score above 3.4 accompanies at least one of the poor prognosis findings (sensitivity $77.6 \%$, specificity $61 \%$ ).

Conclusion: In patients with COVID-19 pneumonia, the presence of at least one of the infection markers that are poor prognosis markers at the time of diagnosis indicate that the modified CT severity score of pneumonia will be above 3.4.

Keywords: COVID-19, pneumonia, tomography, X-ray computed, lymphocyte count, fibrin fragment D, C-reactive protein

\section{Introduction}

The diagnosis of Coronavirus disease-2019 (COVID-19) pneumonia, which is a viral infection, is made by the positivity of the real-time reverse transcriptase-polymerase chain reaction (RT-PCR) test. Since pneumonia findings on computed tomography (CT) can sometimes appear before RT-PCR positivity in the literature, radiology has become important in the diagnosis $(1,2)$, and initiatives have been undertaken to establish a common reporting language to make $\mathrm{CT}$ findings easier to understand by the clinicians (3-5). All of these CT findings, which can be classified as ground-glass opacities, cobblestones, and consolidation were written descriptions of $\mathrm{CT}$ images of COVID pneumonia $(6,7)$.

While at the beginning of the pandemic CT reporting of COVID-19 pneumonia findings was in the form of describing findings, over time, requests began to be made to determine the amount of parenchymal involvement to have an idea about the severity of the disease. In line with similar studies published during the pandemic, we thought that in the evaluation of patients with COVID-19 pneumonia, the pattern and amount of involvement in the lung parenchyma became important $\mathrm{CT}$ findings. Especially, if a relationship could be shown between these $C T$ findings and laboratory findings, the

Address for Correspondence: Ozlem Gungor, University of Health Sciences Turkey, Kecioren

Training and Resarch Hospital, Clinic of Radiology, Ankara, Turkey

Phone: +90 5058750846 E-mail: ozlemkocal@gmail.com ORCID: orcid.org/0000-0003-0793-3498

Received: 19.02.2021 Accepted: 31.03.2021

Copyright 2021 by The Medical Bulletin of istanbul Haseki Training and Research Hospital The Medical Bulletin of Haseki published by Galenos Yayınevi. 
clinician could then predict the severity of pneumonia to be encountered in $C T$, even if $C T$ examination was not performed at certain laboratory values. Therefore, in our study, we developed a new scoring system based on the amount of $\mathrm{CT}$ involvement as well as the involvement pattern (affecting the weight of parameters) and aimed to investigate the relationship between this scoring system and inflammatory markers used by the Ministry of Health as poor prognostic factors. Thus, we planned to show how accurately the poor prognostic factors in the laboratory values of the patient at the time of CT can be identified with this $\mathrm{CT}$ scoring system and to provide the clinician with an idea about the severity of pneumonia with these laboratory findings at the time of seeing the patient.

\section{Methods}

\section{Study Design}

This single-center, a retrospective cross-sectional study was conducted with patients who were diagnosed with COVID-19 pneumonia in a training and research hospital between March and June 2020 after receiving approval from a local ethical committee.

\section{Study Population}

Before starting the study, approval was obtained from the Ethics Committee of Kecioren Training and Research Hospital (10.6.2020/2116). Among the patients diagnosed with COVID-19 with positive RT-PCR test performed on throat swabs between March 17 and May 1, 2020, in our hospital, 89 (male/female: 44/45) patients with typical findings of COVID-19 pneumonia according to the American College of Radiology (ACR) guidelines but did not have any other signs of disease affecting the lung (such as AC malignancy, lobectomy, or tuberculosis) were included in the study. The study was retrospective, so an informed consent form was not applicable.

Neutrophil count, lymphocyte count, serum C-reactive protein value, and D-dimer value were recorded from the examination performed within 24 hours of the CT scan dates of the patients by an infectious diseases expert who was blinded to the $\mathrm{CT}$ findings. Patients were divided into two groups as those with severe infection and those without, according to whether they exhibited poor prognostic criteria specified in the Ministry of Health guideline (Blood lymphocyte count $<800 / \mu \mathrm{L}$ or C-reactive protein $>10 x$ the upper limit of normal value or D-dimer $>1000 \mathrm{ng} / \mathrm{mL}$ ) (8). The CT findings and scores described below were compared between these two groups of patients.

\section{Computed Tomography}

All CT examinations were performed by a multi-slice $\mathrm{CT}$ device with 16 detectors (Siemens Somatom Emotion
16, Siemens) using automatic dose modulation technique and the same acquisition protocol. Intravenous contrast material was not used in the examination. While in the supine position, the patient was instructed to hold his breath at the end of inspiration, and the examination area was adjusted from the lung apex to the end of the costophrenic angle. CT settings were as follows: $120 \mathrm{kVp}$, 1.35:1 pitch, reconstruction matrix $512 \times 512$, high-spatialresolution algorithm, and $1 \mathrm{~mm}$ section thickness. Images were analyzed in three planes using the multiformat imaging technique.

\section{Computed Tomography Evaluation}

All CT images were retrospectively evaluated in the lung window (WW: $1500 \mathrm{HU}, \mathrm{WL}:-500 \mathrm{HU}$ ) by two expert radiologists (OG; $\mathrm{CO})$ with 13 and 12 years of experience in thoracic radiology. Lung parenchymal involvement findings were named according to the definitions in the ACR guideline (4). The severity of pulmonary parenchymal findings was scored using two separate scoring systems previously described in the literature $(9,10)$, as well as a third separate scoring system, namely the "modified CT severity scoring" defined below. There was a two-week gap between the evaluations made with these three separate scoring systems. These scoring systems were as follows:

1. CT score: Developed by Pan et al. (9), this scoring was made according to the percentage of involvement in each lobe. The scores obtained for each lobe $(0=a b s e n t$, $1=1-5 \%$ involvement rate, $2=6-25 \%$ involvement rate, $3=26-50 \%$ involvement rate, $4=51-75 \%$ involvement rate, $5=>75 \%$ involvement rate) were summed up to obtain the "total lung involvement score". In this system, the minimum and the maximum score for each case were 0 and 25 , respectively [5 (lobe) $\times 5$ (involvement rate)].

2. CT severity score: This scoring system was developed by Huang et al. (10). In this system, a score was obtained for each lobe ( $0=$ absent, $1=1-5 \%$ involvement rate, $2=6$ $25 \%$ involvement rate, $3=26-50 \%$ involvement rate, $4=51-75 \%$ involvement rate, $5=>75 \%$ involvement rate) and then "1" was added to this score if cobblestone appearance was present or "2" was added to this score if consolidation was present in one lobe. The "total lung involvement score" was found by summing the scores of all lobes. In this system, the minimum and maximum scores for each case were determined as 0 and 35 [5 (lobe) $\times 5$ (involvement ratio) + weight coefficient].

3. Modified CT severity score: 18 lung segments in total in two lungs were divided into 20 regions. The left lung apicoposterior segment was divided into apical and posterior regions, and the left lung anteromedial basal segment was divided into anterior and mediobasal segments. The involvement rates were initially examined 
for the findings in each region ( $0=$ absent, $1=1-49 \%$ involvement rate, $2=50-100 \%$ involvement rate). Parenchymal findings in each region were divided into five according to ground-glass opacities, a cobblestone appearance, mixed type with predominantly ground-glass opacities, mixed type with predominantly consolidation, and pure consolidation. Among these parenchymal findings, involvement coefficients were formed by separating the "involvement feature" into further groups (ground-glass opacities "0.2"; cobblestone appearance "0.4"; mixed type with predominantly ground glass opacities "0.6", mixed type with predominantly consolidation " 0.8 " and pure consolidation "1"). The involvement score of each region was obtained by multiplying the involvement coefficient and ratio for each region. The sum of the involvement scores of the 20 regions gave the "total lung involvement score". In this system, the minimum and the maximum score for each case was determined as 0 and 40 [20 regions $x^{2}$ (involvement ratio) x1 (involvement feature)].

\section{Statistical Analysis}

All data were analyzed by IBM SPSS Statistics for Mac, version 25.0 for Mac OS X (IBM Corp., Armonk, N.Y., USA). The categorical values of the patients were expressed as a number and a percentage and were analyzed with a chisquare test. Whether the numerical variables were normally distributed or not was evaluated with the Shapiro-Wilk test, histogram, and Q-Q plots. While normally distributed numeric variables were presented as a mean and standard deviation, non-parametric variables were presented as median values and an interquartile range (IQR) of $25 \%$ $75 \%$. The non-parametric values were analyzed using the Mann-Whitney $U$, and the parametric ones with a Student's t-test. To assess the prognostic utility of CT scores at varying cut-off values for the distinction between the severe and non-severe infection groups, a receiveroperating characteristic (ROC) curve was generated, and the area under the curve (AUC) was calculated (Figure 1). The best of cut-off values was decided by using Youden's index. The 95\% confidence intervals (95\% Cls) were calculated whenever appropriate, and a two-tailed $p<0.05$ was considered statistically significant.

\section{Results}

The mean age of patients was $49 \pm 14.2$ and 44 $(49.2 \%)$ of them were male. Thirty (33.7\%) patients were categorized in the severe infection group and fifty-nine $(66.3 \%)$ of them were categorized with the non-severe infection group according to laboratory examination. In comparing CT findings and CT scores of both groups, it was found that all CT score points were higher in the severe infection group than the non-severe infection group $(p<0.05)$. All CT scores and laboratory results were presented in Table 1.

To assess the prognostic utility of all CT scores at varying cut-off values for the distinction between the severe and non-severe infection groups, a ROC curve was generated, and the AUC was calculated. Accordingly, the AUC values of CT score, CT severity score and modified CT severity score 0.711 (95\% Cl: 0.592 to 0.830$), 0.684$ (95\% Cl: 0.564 to $0.804)$ and 0.722 (95\% Cl: 0.615 to 0.829$)$, respectively. The best cut-off value of all CT scores for distinguishing between the severe and non-severe infection groups and the sensitivity/specificity values for this cut-off level were presented in Table 2. Also, for modified CT score,

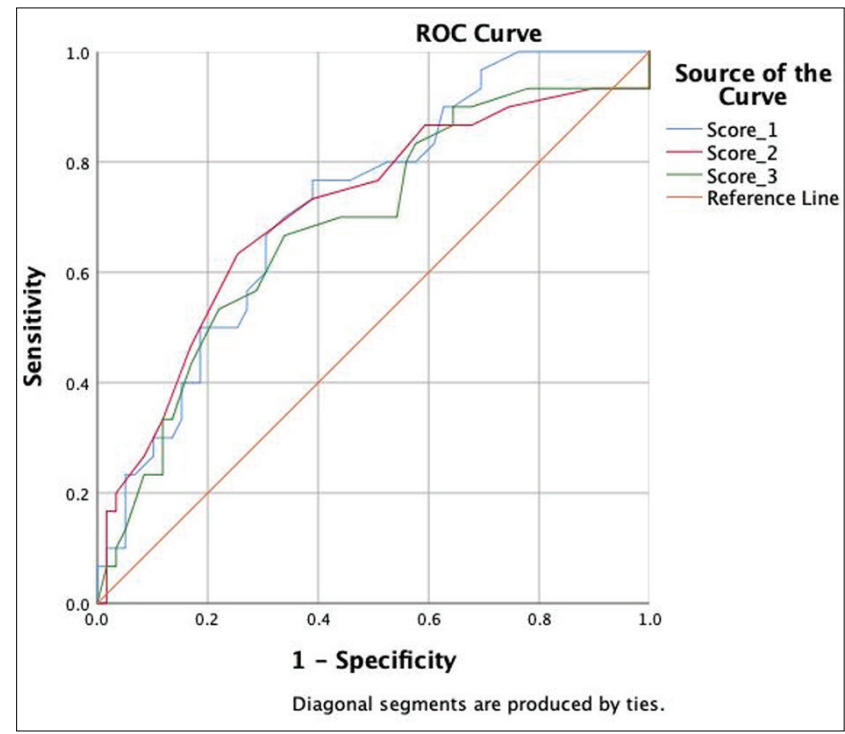

Figure 1. ROC analysis data

ROC: Receiver-operating characteristic

\begin{tabular}{|c|c|c|c|}
\hline $\begin{array}{l}\text { Final CT } \\
\text { scores }\end{array}$ & $\begin{array}{l}\text { Non-Severe } \\
\mathrm{n}=59\end{array}$ & $\begin{array}{l}\text { Severe } \\
\mathrm{n}=30\end{array}$ & p \\
\hline CT score & 7 (3 to 9$)$ & $9(6.75$ to 12$)$ & 0.001 \\
\hline $\begin{array}{l}\text { CT severity } \\
\text { score }\end{array}$ & $11(5$ to 14$)$ & 15 (10 to 18.75$)$ & 0.005 \\
\hline $\begin{array}{l}\text { Modified } \\
\text { CT severity } \\
\text { score }\end{array}$ & $3(1.4$ to 5.4$)$ & 5.5 (3.3 to 8.7$)$ & 0.001 \\
\hline \multicolumn{4}{|c|}{ Laboratory findings on admission median (IQR $25-75 \%$ ) } \\
\hline Neutrophil & 3470 (2810 to 4160$)$ & 4150 (3345 to 5165$)$ & 0.013 \\
\hline Lymphocyte & 1720 (1320 to 2220$)$ & 1090 (740 to 1705$)$ & $<0.001$ \\
\hline D-dimer & 370 (280 to 540$)$ & 680 (330 to 1580$)$ & 0.003 \\
\hline $\begin{array}{l}\text { C-reactive } \\
\text { protein }\end{array}$ & 8.3 (3.9 to 19$)$ & 35.6 (7.25 to 65.7$)$ & $<0.001$ \\
\hline
\end{tabular}


diagnostic performance values and Youden's index scores for different cut-off points were presented in Table 3. According to this, 3.4 points were considered as a cut-off, sensitivity/specificity values of modified CT severity score for the diagnosis of severe COVID-19 pneumonia were as follows; 76.7 (95\% Cl: 57.72 to 90.07) and 61 (95\% Cl: 47.44 to 73.45$)$, respectively.

\section{Discussion}

Even though it feels like COVID-19 has been in our personal lives for a lifetime, COVID-19 is an infection that we have been faced with for a short time scientifically and therefore a lot about it is still unknown. Although certain progress has been made in terms of diagnosis, patient management and treatment strategies vary. Since it is a pandemic, the fact that many people are infected at the same time has made patient management more important. In Turkey, treatment and patient management are standardized according to the guidelines set by the Ministry of Health (8). In the present study, we found that in cases where at least one of the poor prognostic markers specified in this guideline was high (high C-reactive protein, high D-dimer, and lymphopenia), the "modified CT severity score" was also high $(p<0.01)$.

Different laboratory data were used in studies trying to predict clinical severity in COVID-19 patients. As in our study, "CT score," which is the first scoring system we used in this article, was used in the study conducted by $\mathrm{Li}$ et al. (11) with 90 patients, comparing patients based on infection markers. In this study, CT and C-reactive protein value were higher and lymphocyte count was lower in patients with a severe condition (meeting at least one of the following conditions: respiratory rate 30 times/min, $\mathrm{O}_{2}$ saturation $\leq 93 \%,\left(\mathrm{PaO}_{2}\right) /\left(\mathrm{FiO}_{2}\right) \leq 300$, need for mechanical intubation, shock, and organ failure) $(p<0.001)$. Francone et al. (12) used the same scoring system and found a statistically significant positive correlation between the CT score and C-reactive protein $(r=0.6204, p<0.0001)$ and D-dimer $(r=0.6625, p<0.0001)$. In the present study, in all three scoring systems used in parallel with these findings, we found that the scores were higher in terms of infection parameters in the presence of poor prognosis, and the most significant results were obtained with the "modified CT score" system $(p<0.001)$.

It was understood that lung involvement was the basis of the events affecting the poor prognosis of the patients during the pandemic. During the SARS epidemic, Chang et al. (13) showed that beyond the presence of lung involvement, the amount of involvement in viral infections was also important in patient management (13). Based on this scoring system, Pan et al. (9) created the "CT score" that takes into account the involvement rates in the lungs (0-5-25-50-75-100\%) of patients with COVID-19 pneumonia. In this study involving 21 cases, patients were divided into 4 stages according to the time between symptom onset and CT scan, and CT score was found to be higher in those with a longer-term disease history [stage 1 (0-4 days) CT score $2 \pm 2$, stage 2 (5-8 days) CT score $6 \pm 4$, stage 3 (9-13 days) CT score $7 \pm 4$, stage 4 (>14 days) CT score $6 \pm 4$ ]. In the same study, the most common finding was ground-glass opacity for stages 1 and 2, and consolidation for stages 3 and 4 . In the present study, we

Table 2. The prognostic values of all CT scores to the prediction of severe infection in patients with diagnosed COVID-19 pneumonia

\begin{tabular}{|l|l|l|l|}
\hline Variables & CT score & CT severity score & Modified CT severity score \\
\hline AUC $(95 \% \mathrm{Cl})$ & $0.711(0.592$ to 0.830$)$ & $0.684(0.564$ to 0.804$)$ & 0.722 (0.615 to 0.829$)$ \\
\hline Best cut-off value* & 9 & 13 & 3.4 \\
\hline Sensitivity $(95 \% \mathrm{Cl})$ & $63.33(43.86$ to 80.07$)$ & $66.67(47.19$ to 82.71$)$ & $76.7(57.72$ to 90.07$)$ \\
\hline Specificity $(95 \% \mathrm{Cl})$ & $74.58(61.56$ to 85.02$)$ & $66.1(52.61$ to 77.92$)$ & $61(47.44$ to 73.45$)$ \\
\hline PLR $(95 \% \mathrm{Cl})$ & $2.49(1.49$ to 4.17$)$ & $1.97(1.27$ to 3.05$)$ & $1.97(1.35$ to 2.87$)$ \\
\hline NLR $(95 \% \mathrm{Cl})$ & $0.49(0.3$ to 0.8$)$ & $0.5(0.29$ to 0.86$)$ & $0.38(0.19$ to 0.75$)$ \\
\hline Accuracy $(95 \% \mathrm{Cl})$ & $70.79(60.19$ to 79.95$)$ & $66.29(55.49$ to 75.97$)$ & $66.29(55.49$ to 75.97$)$ \\
\hline $\begin{array}{l}\text { *The best of cut-off values was decided by using Youden's index. AUC: Area under curve, PLR: Positive likelihood ratio NLR: Negative likelihood ratio, COVID-19: Coronavirus } \\
\text { disease-2019, CT: Computed tomography, IQR: Interquartile range, Cl: Confidence intervals }\end{array}$ &
\end{tabular}

Table 3. The sensitivity and specificity values for different cut-off points of modified CT severity score

\begin{tabular}{|l|l|l|l|l|l|l|}
\hline $\begin{array}{l}\text { Modified CT } \\
\text { score }\end{array}$ & Sensitivity (\%) & Specificity (\%) & NLR & PLR & Accuracy & $\begin{array}{l}\text { Youden's } \\
\text { index }\end{array}$ \\
\hline 2 & $90(73$ to 97$)$ & $35(23$ to 49$)$ & $0.28(0.09$ to 0.86$)$ & $1.4(1.1$ to 1.75$)$ & $53(43$ to 64$)$ & 0.255 \\
3 & $80(61$ to 92$)$ & $47(34$ to 60$)$ & $0.42(0.2$ to 0.9$)$ & $1.5(1.1$ to 2.05$)$ & $58(47$ to 68$)$ & 0.274 \\
3.4 & $76(57$ to 90$)$ & $61(47$ to 73$)$ & $0.38(0.19$ to 0.75$)$ & $1.9(1.35$ to 2.8$)$ & $66(55$ to 75$)$ & 0.376 \\
5 & $53(34$ to 71$)$ & $72(59$ to 83$)$ & $0.64(0.42$ to 0.97$)$ & $1.9(1.1$ to 3.3$)$ & $66(55$ to 75$)$ & 0.262 \\
6 & $50(31$ to 68$)$ & $81(69$ to 90$)$ & $0.61(0.42$ to 0.89$)$ & $2.6(1.4$ to 5.09$)$ & $70(60$ to 79$)$ & 0.313 \\
\hline
\end{tabular}


found that all scoring systems, including the CT score, were successful in detecting severe infection $(p<0.001)$.

We stated that we named the systems in the literature in which the involvement pattern is also included in the scoring as "CT severity score". Using the "CT severity score", Yuan et al. (14) conducted a study with 27 patients (a coefficient of 2 for ground glass and 3 for consolidation was used) and found that the median CT score of the cases that resulted in mortality was higher than the surviving group [(30 (IQR) 7-13) vs 12 (IQR 11-43), $\mathrm{p}=0.021]$. This study, unlike many other studies, divided the lungs into only three zones. In the present study, we divided and evaluated the lung in 20 regions, ensuring that the evaluation included as complete information on the lung as possible, and included 5 different involvement categories into the evaluation, enabling the severity of infection to be included in the scoring according to the histopathological response in the patient.

The basis of this was that the findings we saw on CT showed different processes histopathologically. Ground glass appearance is defined as images caused by pulmonary edema and hyaline membrane formation, cobblestone appearance is defined as images caused by alveolar edema and interstitial inflammation, and consolidation is defined as images caused by cellular fibromyxoid exudate accumulation in the alveoli $(1,15)$. We have created a formula in which the area of involvement in each lung area is more effective, but the pattern of this involvement is also considered. While the mean score was 3 (1.4-5.4) in our non-severe patient group, the mean score was 5.5 (3.3-8.7) in the severe group, and the difference between the groups was significant $(p<0.001)$. We think that the reason for the relatively low scores in our patient groups is that the patients presented at an early stage and time of their complaints. Since we aim to show the relationship between infection markers at the time of diagnosis and a recent $\mathrm{CT}$ score, the low maximum score indicates that the participants were in the early period of the disease.

We determined that this scoring system, in which histopathological information has been added to the CT information with the created "modified CT severity score", was better correlated with the elevation in at least one of the infection markers, which were defined as poor prognosis indicators in the literature, compared with the other scoring systems described previously (Table 2). Accordingly, considering the cut-off point obtained with the modified CT score, this cut-off value can be used as a support parameter to indicate poor prognosis of the disease, or if the specified limits are exceeded ven in one of the parameters such as lymphocyte, C-reactive protein, and D-dimer that can be checked in many countries around the world, time of the CT scan can be changed with the prediction that the patient's pneumonia may be acute or this information can support disease management in cases where CT cannot be performed.

\section{Study Limitations}

There are certain limitations of this study. Clinical outcome was not evaluated in our study. It is known that clinical outcome is the result of multiple factors such as age, comorbidity, time of treatment initiation, and the applied treatment protocol $(14,16-20)$. The objective of this study was to evaluate the correlation of CT findings at the time of diagnosis with the laboratory values obtained at the same time and to support the clinician's management of newly diagnosed patients for COVID-19 pneumonia based on our inferences. Another limitation is that the number of patients in our study was 89 , but statistical significance could be detected for scoring. There is a need for further studies involving larger patient groups to validate our findings obtained with the modified CT score. Another limitation may be the lack of artificial intelligence used to evaluate CT in this study. However, we tried to establish a scoring system that can be used all over the world during a pandemic, and we thought that it would best that this scoring system did not rely on high-priced technology.

\section{Conclusion}

If there is at least one of the poor prognosis findings (high C-reactive protein, high D-dimer, and lymphopenia) in the infection markers of cases with COVID-19 pneumonia, pneumonia will accompany on $\mathrm{CT}$ with a modified $\mathrm{CT}$ score above 3.4 (sensitivity $76 \%$, specificity $61 \%$ ). For this reason, these markers exceeding the specified limits should make the clinician think that pneumonic infiltration becomes prominent on $\mathrm{CT}$ (hence the modified CT severity score increases). We believe that this cut-off point can be used in addition to laboratory data as a criterion for hospitalization, or patient treatment can be guided based on laboratory findings in cases where CT cannot be performed.

\section{Authorship Contributions}

Concept: O.G., C.O., S.U.R., Design: O.G., C.O., S.U.R., Data Collection or Processing: O.G., Z.N.S., A.B.Y., Analysis or Interpretation: O.G., S.U.R., S.K.C., Literature Search: O.G., C.O., S.U.R., Writing: O.G., S.U.R.

Conflict of Interest: No conflict of interest was declared by the authors.

Financial Disclosure: The authors declared that this study received no financial support.

\section{References}

1. Ye Z, Zhang $Y$, Wang $Y$, Huang Z, Song B. Chest CT manifestations of new coronavirus disease 2019 (COVID-19): a pictorial review. Eur Radiol 2020;30:4381-9. 
2. Zhao W, Zhong Z, Xie X, Yu Q, Liu J. Relation Between Chest CT Findings and Clinical Conditions of Coronavirus Disease (COVID-19) Pneumonia: A Multicenter Study. AJR Am J Roentgenol 2020;214:1072-7.

3. Rubin GD, Ryerson CJ, Haramati LB, et al. The Role of Chest Imaging in Patient Management during the COVID-19 Pandemic: A Multinational Consensus Statement from the Fleischner Society. Radiology 2020;296:172-80.

4. Lang M, Som A, Mendoza DP, et al. Detection of Unsuspected Coronavirus Disease 2019 Cases by Computed Tomography and Retrospective Implementation of the Radiological Society of North America/Society of Thoracic Radiology/American College of Radiology Consensus Guidelines. J Thorac Imaging 2020 Jun 17.

5. Vogel-Claussen J, Ley-Zaporozhan J, Agarwal P, et al. Recommendations of the Thoracic Imaging Section of the German Radiological Society for clinical application of chest imaging and structured CT reporting in the COVID-19 pandemic. Rofo 2020;192:633-40.

6. Zhou S, Wang Y, Zhu T, Xia L. CT Features of Coronavirus Disease 2019 (COVID-19) Pneumonia in 62 Patients in Wuhan, China. AJR Am J Roentgenol 2020;214:1287-94.

7. Bernheim $A$, Mei $X$, Huang $M$, et al. Chest $C T$ Findings in Coronavirus Disease-19 (COVID-19): Relationship to Duration of Infection. Radiology 2020;295:200463.

8. Republic of Turkey Ministry of Health Treatment guidelines for adult patients Covid-19 (SARS-CoV2 infection. Published 2020 Aug 2 . https://covid19.saglik.gov.tr/TR-66926/eriskinhasta-tedavisi.html\#

9. Pan F, Ye T, Sun P, et al. Time Course of Lung Changes at Chest CT during Recovery from Coronavirus Disease 2019 (COVID-19). Radiology 2020;295:715-21.

10. Huang G, Gong T, Wang G, et al. Timely Diagnosis and Treatment Shortens the Time to Resolution of Coronavirus Disease (COVID-19) Pneumonia and Lowers the Highest and Last CT Scores From Sequential Chest CT. AJR Am J Roentgenol 2020;215:367-73.
11. Li K, Wu J, Wu F, et al. The Clinical and Chest CT Features Associated With Severe and Critical COVID-19 Pneumonia. Invest Radiol 2020;55:327-31.

12. Francone $M$, lafrate $F$, Masci $G M$, et al. Chest $C T$ score in COVID-19 patients: correlation with disease severity and short-term prognosis. Eur Radiol 2020;30:6808-17.

13. Chang YC, Yu CJ, Chang SC, et al. Pulmonary sequelae in convalescent patients after severe acute respiratory syndrome: evaluation with thin-section CT. Radiology 2005;236:106775.

14. Yuan M, Yin W, Tao Z, Tan W, Hu Y. Association of radiologic findings with mortality of patients infected with 2019 novel coronavirus in Wuhan, China. PLoS One 2020;15:e0230548.

15. Razek A, Fouda N, Fahmy D, et al. Computed tomography of the chest in patients with COVID-19: what do radiologists want to know? Pol J Radiol 2021;86:122-35.

16. Wu C, Chen X, Cai Y, et al. Risk Factors Associated With Acute Respiratory Distress Syndrome and Death in Patients With Coronavirus Disease 2019 Pneumonia in Wuhan, China. JAMA Intern Med 2020;180:934-43.

17. Liu W, Tao ZW, Wang L, et al. Analysis of factors associated with disease outcomes in hospitalized patients with 2019 novel coronavirus disease. Chin Med J (Engl) 2020;133:10328.

18. Yang $X, Y u Y, X u$ J, et al. Clinical course and outcomes of critically ill patients with SARS-CoV-2 pneumonia in Wuhan, China: a single-centered, retrospective, observational study. Lancet Respir Med 2020;8:475-81.

19. Romeyke T, Noehammer E, Stummer H. COVID-19 Patient with Severe Comorbidity in Multimodal Acute Care Setting with Non-Invasive Medical Ventilation: A Clinical Outcome Report. Clin Pract 2021;11:81-91.

20. Akiyama S, Hamdeh S, Micic D, Sakuraba A. Prevalence and clinical outcomes of COVID-19 in patients with autoimmune diseases: a systematic review and meta-analysis. Ann Rheum Dis 2021;80:384-91. 